九州大学学術情報リポジトリ

Kyushu University Institutional Repository

\title{
Effectiveness of Approximation Strategy in Surrogateassisted Fireworks Algorithm
}

Pei, Yan

Computer Science Division, The University of Aizu

Zheng, Shaoqiu

Department of Machine Intelligence, School of ElectronicsEngineering and Computer Science, Peking University

Tan, Ying

Department of Machine Intelligence, School of ElectronicsEngineering and Computer Science, Peking University

Takagi, Hideyuki

Faculty of Design, Kyushu University

http://hdl. handle. net/2324/1518761

出版情報: International Journal of Machine Learning and Cybernetics. 6 (5), pp.795-810，201507-14. Springer Berlin Heidelberg

バージョン :

権利関係 : 


\title{
Effectiveness of Approximation Strategy in Surrogate- assisted Fireworks Algorithm
}

\author{
Yan Pei · Shaoqiu Zheng • Ying Tan • Hideyuki Takagi
}

Received: date / Accepted: date

\begin{abstract}
We investigate the effectiveness of approximation strategy in a surrogate-assisted fireworks algorithm, which obtains the elite from approximate fitness landscape to enhance its optimization performance. We study the effectiveness of approximation strategy from the aspects of approximation method, sampling data selection method and sampling size. We discuss and analyse the optimization performance of each method. For the approximation method, we use least square approximation, spline interpolation, Newton interpolation, and support vector regression to approximate fitness landscape of fireworks algorithm in projected lower dimensional, original and higher dimensional search space. With regard to the sampling data selection method, we define three approaches, i.e., best sampling method, distance near the best fitness individual sampling method,
\end{abstract}

Yan Pei

Computer Science Division, The University of Aizu, Aizuwakamatsu, 965-8580 Japan

E-mail: peiyan@u-aizu.ac.jp

Homepage: http://web-ext.u-aizu.ac.jp/ peiyan/

Shaoqiu Zheng

Department of Machine Intelligence, School of Electronics Engineering and Computer Science, Peking University

Key Laboratory of Machine Perception (Ministry of Education), Peking University, Beijing, 100871 P.R. China

E-mail: zhengshaoqiu@pku.edu.cn

Ying Tan

Department of Machine Intelligence, School of Electronics Engineering and Computer Science, Peking University

Key Laboratory of Machine Perception (Ministry of Education), Peking University, Beijing, 100871 P.R. China

E-mail: ytan@pku.edu.cn

Hideyuki Takagi

Faculty of Design, Kyushu University, Fukuoka, 815-8540 Japan

E-mail: takagi@design.kyushu-u.ac.jp

Homepage: http://www.design.kyushu-u.ac.jp/ takagi/ and random sampling method to investigate each sampling method's performance. With regard to sample size, this is set as 3, 5, and 10 sampling data in both the approximation method and sampling method. We discuss and compare the optimization performance of each method using statistical tests. The advantages of the fireworks algorithm, a number of open topics, and new discoveries arising from evaluation results, such as multi-production mechanism of the fireworks algorithm, optimization performance of each method, elite rank, interpolation times and extrapolation times of elites are analysed and discussed.

Keywords Fireworks Algorithm - Fitness Landscape Approximation · Elite Strategy · Surrogate-assisted Fireworks Algorithm · Dimensionality Reduction

\section{Introduction}

Evolutionary computation (EC) has demonstrated the powerful capability to solve complex industrial and engineering optimization problems. From a framework viewpoint, there are three parts in an EC based optimization system, which include a target system that should be optimized, an EC algorithm (including an user interface for interactive evolutionary computation (IEC) [21]) that conducts the concrete optimization operations, and one or multiple fitness function(s) (including a human user for IEC). These three parts encompass the corresponding three study aspects of an EC based optimization system, i.e., EC applications, EC algorithms, and EC fitness function(s) or an IEC human user.

The EC algorithm performance is a serious issue for its real application. There are three promising study directions aiming to meet this challenge in recent decades 
within EC community [9]. First is to approximate fitness landscape and make an operation or a model to assist the EC search. Takagi, et. al. proposed to use a single peak function to approximate fitness landscape and conduct a local search by using an elite obtained from approximated landscape [22]. Pei, et. al. extended this work by conducting the approximation in each lower dimensional search space for simplifying the approximation computation [12]. Other approximate methods by obtaining a frequency component information from fitness landscape was proposed in $[11,16]$. Second is to develop effective search strategy or revise search mechanism into an EC algorithm to obtain an accelerated search convergence. Memetic algorithm was proposed to conduct individual learning or local improvement procedures for optimization [7]. It also can be introduced into the IEC for obtain a better optimization performance [15]. A series of search strategies were develope$\mathrm{d}$ in differential evolution (DE) community, which include SaDE [18], jDE [1], SaJADE [2], JADE [25], etc. A triple and quadruple comparison-based DE was proposed in [14], which an opposition-based learning process is embedded into the DE selection process. These novel strategies and mechanisms can be categorized into two aspects, the one is searching in a form of hybrid global evolutionary algorithm coupled with a learning procedure capable of local refinements, the other is conducting the different search strategies according to the knowledge obtained from the search process. Third is to create a new type of EC algorithm that is inspired by biological, physical or mathematical schemes and phenomenon, such as the fireworks algorithm [23], chaotic evolution [8,10], water wave optimization [27], water cycle algorithm [19], optics inspired optimization [6], etc. This is the primary subject of investigation in this paper.

Fireworks algorithm (FWA) is a recently developed algorithm [23]. It is inspired by the explosion of fireworks in the night sky, which can illuminate the dark space. The process of illuminating the nearby space of a firework position can be considered as the search among the feasible range. The FWA work mechanism is described in the following. A number of fireworks are set off to the feasible range, and each position of the firework will be evaluated by the fitness function. The explosion amplitude and spark number for each firework are calculated based on the fitness of the fireworks. For a minimal objective problem, the principle is that the firework with smaller fitness will have a smaller explosion amplitude and a larger number of sparks. The fireworks are explored under the calculated explosion amplitude and spark number. Moreover, the Gaussian explosion sparks are also generated to enhance the di- versity of the FWA. A number of fireworks are selected from the candidate sets, which include the fireworks, regular explosion sparks and Gaussian explosion sparks. The FWA will continue until the termination condition is met.

We proposed to use a surrogate model for improving the performance of FWA. Surrogate-assisted FWA uses efficient computational models for approximating the fitness landscape where we obtain the elite from search space to accelerate FWA search. The method is to approximate the fitness landscape in the one-dimensional search space, and obtain and synthesize the elite from the approximated landscape curves. When the elite is better than the worst firework, surrogate-assisted FWA replaces the worst one with this elite into the next generation to enhance the FWA search. An empirical study on influence of approximation methods on the FWA acceleration performance was initially investigated and analysed [17]. However, some of the problems in applying this method need further study and investigation.

This paper extends the work of [17] and conducts a further investigation on the approximation method and strategy influence on surrogate-assisted FWA. We especially investigate: (1) several approximation methods for approximating fitness landscape and their acceleration performance, (2) sampling methods and sizes' influence on acceleration performance. (3) the obtained elite rank in the population and its effectiveness. (4) the obtained elite from within or beyond the approximation interval. (5) statistical test on the proposed methods' significance. Several related issues are also investigated and discussed. The evaluation metrics include the convergent fitness value after the same number of generations, Wilcoxon signed-rank test, elite rank, and the number of interpolation and extrapolation, etc.

Following this introductory section, in section 2 , an overview on the FWA is presented. The history, inspiration and development of the FWA are described in detail. In section 3, we introduce several techniques on fitness landscape approximation and theoretically discuss the computational complexity. The surrogate-assisted FWA enabled by a technique for reducing dimensionality of the search space is explained, and we show how elite can be obtained from the regression search space. The fitness landscape can be approximated in lower dimensional space, original space and higher dimensional space. In section 4, experimental evaluations with 25 benchmark functions with 10 dimension (10-D) and 30$\mathrm{D}$ are conducted, and their results are analysed and discussed. Finally, we discuss our proposed methods and obtained results in section 5, and conclude the analysis and investigation results of the proposed methods, 
present future opportunities and open topics in section 6.

\section{An Overview of the Fireworks Algorithm}

As one of the swarm intelligence algorithms, the FWA is inspired by the phenomenon of the explosion of fireworks in the night sky. When a firework explodes in the night sky, it illuminates the dark space, which can be considered as a kind of search process. In the FWA, the fireworks generate sparks candidates around themselves in search space. The search operators, which maintain the exploration ability for the heuristic algorithms, can be implemented by simulating some fireworks' explosions in the potential space.

There are three promising research aspects in recent studies of the FWA. The first one is to construc$\mathrm{t}$ a hybrid optimization framework by using the FWA with other EC algorithms or computational mechanisms. Zheng et. al. proposed a hybrid FWA with biogeographybased optimization to enhance the optimization performance of canonical FWA [24]. Ding et. al. implemented a parallel FWA by using a parallel processing architecture, GPU. The second aspect is to establish a FWA optimization framework to solve multi-objective optimization problems [28], constrained optimization problems, combinatorial optimization problems, etc. The third aspect is to apply the FWA to the real-world applications. Janecek et. al. used the FWA with other EC algorithms, such as particle swarm optimization, genetic algorithms, differential evolution, and fish school search, for improving the initialization of the non-negative matrix factorization problem [4].

In general, given the following single objective function $\left\{\operatorname{minf}: \Omega \subseteq R^{n} \rightarrow R\right\}$, the FWA is to find a point $x \in \Omega$, for which $\mathrm{x}$ has the minimal value. Algorithm 1 presents the framework of FWA. In each generation of FWA, $N$ fireworks are randomly initialized in the feasible search range, and the fitness of each firework will be evaluated to determine the explosion amplitude and explosion sparks number. The basic principle for the explosion amplitude and sparks number is that the firework with better fitness will have smaller explosion amplitude and larger population of sparks to increase the exploited ability, while the firework with worse fitness will have a small population of explosion sparks and large explosion amplitude to maintain search ability. After the calculation of the explosion sparks number and explosion amplitude for each firework, the FWA performs the explosion process to generate the regular explosion sparks by Algorithm 2. To increase the diversity of the FWA, it introduces another sparks named
Gaussian sparks generated by Gaussian mutation operation (Algorithm 3). The selection operator is performed to cause the $N$ fireworks from the candidate set which includes regular sparks, $\hat{m}$ Gaussian explosion fireworks and $N$ fireworks to remain. The algorith$\mathrm{m}$ continues until the termination criterion is met, i.e., maximum generation or evaluation, maximum running time, or the optimum is found.

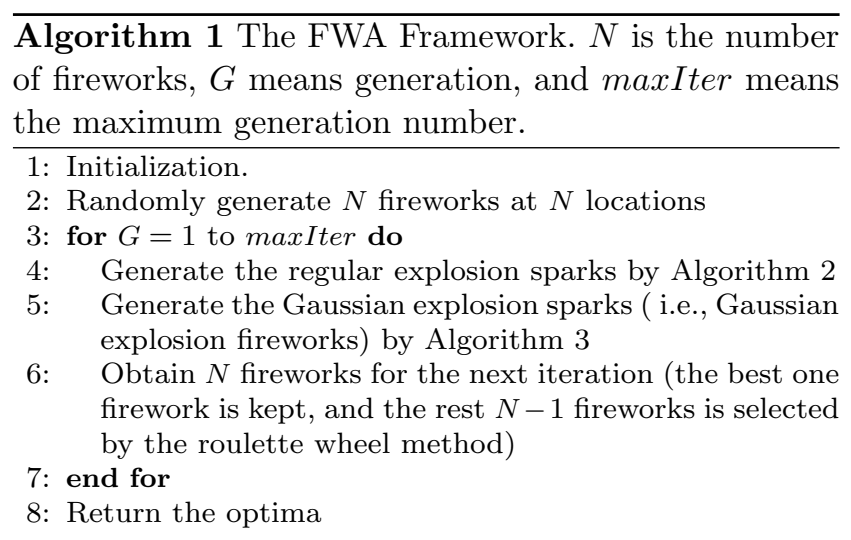

\subsection{Regular Explosion Sparks}

Regular explosion operator is one of the crucial operators in the FWA. For the simulation of the fireworks explosion process, the FWA generates a number of sparks $s_{i}$ within the explosion amplitude $A_{i}$ by Eq.s (1) and (2), where $y_{\max }=\max \left(f\left(x_{i}\right)\right)$ and $y_{\min }=\min \left(f\left(x_{i}\right)\right)$, $i=1,2, \ldots N$. Here $\hat{A}$ and $M$ are constants which are determined by a practical optimization problem. After the calculation of the number of sparks and explosion amplitude, the fireworks perform the generation of the regular explosion sparks by Algorithm 2.

$$
\begin{aligned}
& s_{i}=M \cdot \frac{y_{\max }-f\left(x_{i}\right)+\varepsilon}{\sum_{i=1}^{n}\left(y_{\max }-f\left(x_{i}\right)\right)+\varepsilon} \\
& A_{i}=\hat{A} \cdot \frac{f\left(x_{i}\right)-y_{\min }+\varepsilon}{\sum_{i=1}^{n}\left(f\left(x_{i}\right)-y_{\min }\right)+\varepsilon}
\end{aligned}
$$

\subsection{Gaussian Explosion Sparks}

To increase the diversity of the explosion sparks, another kind of sparks is introduced, named Gaussian explosion sparks. The generation principle of the Gaussian explosion sparks is to create the fireworks by a random value from a Gaussian distribution. The detail of this process is implemented by Algorithm 3 . 


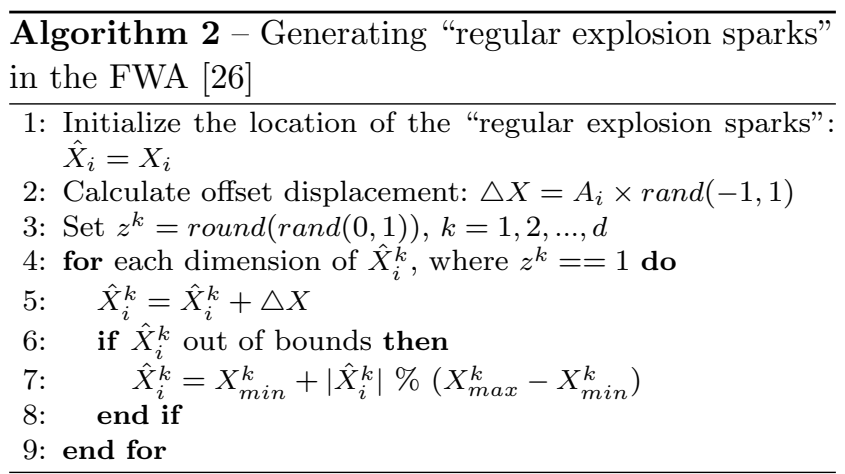

\subsection{Selection for the Next Generation}

After the explosion of fireworks, a number of sparks (regular explosion sparks and Gaussian explosion sparks) are generated. In the selection of fireworks for the next iteration, the candidate (fireworks or sparks) with the best fitness is always kept, the others are selected by the roulette wheel method by using the following Eq.s (3) and (4), where the location $X_{i}$, the selection probability $p\left(X_{i}\right)$ is calculated. Here, $K$ denotes the set of all current locations which includes the fireworks, regular explosion sparks and Gaussian explosion sparks (without the best candidate).

$p\left(X_{i}\right)=\frac{R\left(X_{i}\right)}{\sum_{j \in K} R\left(X_{j}\right)}$
$R\left(X_{i}\right)=\sum_{j \in K} d\left(X_{i}, X_{j}\right)=\sum_{j \in K}\left\|X_{i}-X_{j}\right\|$

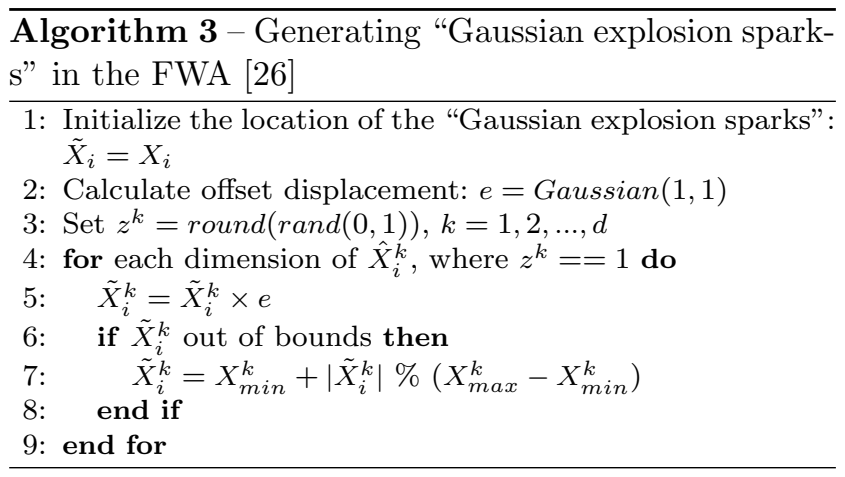

\section{Surrogate-assisted Fireworks Algorithm Framework}

\subsection{Motivation}

Fitness and Fitness landscape approximations are wellknown assisted acceleration techniques in the EC community over the last decade [5]. In some applications, the computation of fitness is time-consuming, so fitness approximation can dramatically save the computation time so as to improve EC performance. On the other hand, fitness landscape approximation can obtain the whole search space structure information to assist EC search. Unlike the conventional EC algorithms, they use the least search information that is supported by the limited individuals' fitness. The computation of either fitness or fitness landscape approximations increases an additional time cost in the EC optimization process. Some of the conventional approximation methods are time-consuming, so reducing the approximation time and developing an efficient approximation method is a promising subject to improve optimization performance of the surrogate-assisted EC.

Approximation method selection and data sampling technique are important issues in the computation of fitness landscape approximation. We have obtained some empirical results on the surrogate-assisted FWA in [17] that shows: (1) The elite strategy is an efficient method to enhance the FWA search capability significantly. (2) The sampling method cannot take effect in isolation, it must be with a proper approximation model to accelerate the FWA search for a certain benchmark function, i.e., a certain fitness landscape. (3) For some benchmark problems, the best sampling method and the random sampling method have the same acceleration performance. (4) The surrogate-assisted FWA can be obtained by a fitness landscape approximation with more sampling size and a proper approximation method. In our study, the better approximation method is the nonlinear model. (5) From a practical point of view, the random sampling method is a better sampling way to obtain the higher acceleration performance in both computational time and final solution quality. However, there are many remaining issues that we need to further investigate, such as the approximate method, the sampling method and the sampling size. These investigations and analyses are one of original features of this paper.

\subsection{Approximation Methods}

In this work, we use the following approximation methods to investigate our proposed acceleration performance influenced by the approximation method. They are least square approximation, spline interpolation, Newton interpolation, and support vector regression

\subsubsection{Least Square Approximation}

The approximation method does not require approximated curves to pass through all discrete points exactly, but rather to approach the original curve at its discrete 
points, i.e. $\left(x_{i}, y_{i}\right)$, as near as possible. When we define the error vector norm as 2-norm, the approximation method is referred to as the least squares method.

There is a function spanning space shown in Eq. (5), $\Phi$ denotes the function class, Span means spanning space, and $\varphi_{i}(x),(i=0,1, \ldots, n)$ denotes a function in the space. We want to find a function that makes the 2-norm error vector $\left(\left\|\delta^{*}\right\|_{2}^{2}\right)$ be minimized as shown in Eq. (6). The approximation function is shown in Eq. (7), where $a_{i}^{*},(i=0,1, \ldots, n)$ are the parameters that make the Eq. (6) to be minimized. If we set $\varphi_{0}(x)=1, \varphi_{1}(x)=x$, and $\varphi_{0}(x)=1, \varphi_{1}(x)=$ $x, \varphi_{2}(x)=x^{2}$ as the approximation function in Eq. (6), they are called linear least squares approximation method and 2-degree polynomial least squares approximation method, respectively.

$$
\begin{aligned}
& \Phi=\operatorname{Span}\left\{\varphi_{0}(x), \varphi_{1}(x), \ldots, \varphi_{n}(x)\right\} \\
& \left\|\delta^{*}\right\|_{2}^{2}=\sum_{i=0}^{m} \delta_{i}^{* 2}=\sum_{i=0}^{m}\left[\varphi^{*}\left(x_{i}\right)-y_{i}\right]^{2}=\min _{\varphi(x) \in \Phi}\left\|\delta^{*}\right\|_{2}^{2} \\
& \varphi^{*}(x)=a_{0}^{*} \varphi_{0}(x)+a_{1}^{*} \varphi_{1}(x)+\ldots+a_{n}^{*} \varphi_{n}(x)
\end{aligned}
$$

\subsubsection{Spline Interpolation}

Spline interpolation is defined by a polynomial function. Compared with polynomial interpolation, spline interpolation has a better approximation performance. We can obtain the interpolation results by spline interpolation with lower degree function, while avoiding the instability of interpolation due to Runge's phenomenon. This shows the advantage of spline interpolation [3].

Suppose that there are different points $\left(x_{i}, y_{i}\right),(i=$ $0,1, \ldots, n)$, the objective of spline interpolation finds an $n$-degree spline function $S(x)$ defined in Eq. (8), where $S_{i}(x)$ is a $k$-degree polynomial function. The linear spline function is given as in Eq. (9).

$S(x)= \begin{cases}S_{0}(x) & x \in\left[x_{0}, x_{1}\right] \\ S_{1}(x) & x \in\left[x_{1}, x_{2}\right] \\ \vdots & \vdots \\ S_{n-1}(x) & x \in\left[x_{n-1}, x_{n}\right]\end{cases}$

$S_{i}(x)=y_{i}+\frac{y_{i+1}-y_{i}}{x_{i+1}-x_{i}}\left(x-x_{i}\right)$

\subsubsection{Lagrangian Interpolation and Newton Interpolation}

The Lagrange interpolation polynomial has the characteristics of linear and unique. For one-dimensional data from individuals $x_{0}, x_{1}, \ldots, x_{n}$, we can set up an $n$ degree polynomial $l_{0}(x), l_{1}(x), \ldots, l_{n}(x)$. We set its type as $l_{i}\left(x_{j}\right)=\delta_{i j}$, where the form of $\delta_{i j}$ is as shown in Eq. (10).

$\delta_{i j}=\left\{\begin{array}{l}0 \text { if } i=j \\ 1 \text { if } i \neq j\end{array}\right.$

From the definition of $l(x)$, we can obtain Eq. (11), which is the $n$-degree interpolation polynomial, where $l_{k}(x)$ is an $n$-degree polynomial. The relationships of the Lagrange interpolation polynomial are shown in Eq.s (11), (12), (13) and (14).

$p_{n}(x)=\sum_{k=0}^{n} l_{k}(x) y_{k}=y_{i}$
$l_{k}(x)=a\left(x-x_{0}\right) \ldots\left(x-x_{k-1}\right)\left(x-x_{k+1}\right) \ldots\left(x-x_{n}\right)(12$

When the condition is $l_{k}\left(x_{k}\right)=1$ in Eq. (12), we can obtain Eq. (13). Then, Eq. (12) can be re-written as in Eq. (14). Eq.s (14) and (15) are the $n$-degree Lagrange interpolation basis function and the $n$-degree Lagrange interpolation polynomial, respectively.

$a=\left[\left(x_{k}-x_{0}\right) \ldots\left(x_{k}-x_{k-1}\right)\left(x_{k}-x_{k+1}\right) \ldots\left(x_{k}-x_{n}\right)\right]^{-1}(13)$

$l_{k}(x)=\prod_{i=1, i \neq k}^{n} \frac{\left(x-x_{i}\right)}{\left(x_{k}-x_{i}\right)}$

$L_{n}(x)=\sum_{k=0}^{n} l_{k}(x) y_{k}, i=0,1, \ldots, n$

Because the polynomial interpolation is unique, the Newton interpolation has the same form as the Lagrange interpolation shown in Eq. (15).

\subsubsection{Support Vector Regression}

Support vector regression (SVR) is a kernel method that is used in regression. If a problem is non-linear, instead of trying to fit a non-linear model, the SVR projects the problem from the input space to a higher dimensional space, i.e. a feature space, to find a linear model by conducting a non-linear transformation using suitably chosen kernel functions. It uses the linear model in the feature space to solve the problem. There are two aspects in the primary motivation of SVR. One is to project the original space into a high dimensional space to find the linearity in such space. The other is to conduct the regression process with those linearity characteristics.

There are several training sample data in the original space, $x_{1}, x_{2}, \ldots, x_{N}$, and there is a feature map function $\phi\left(x_{i}\right), i=1,2, \ldots, N$, which conducts the feature map to project the data into a higher dimensional space (Eq.s (16), (17) and (18), $X$ denotes a matrix that encompasses all the data that are projected into 
a feature space, $y$ denotes the matrix that the value of each data $x_{i}, i=1, \ldots, n$.). From the normal equation of linear regression, we can obtain the support vector regression expression (Eq. (19)). $K(x, y)$ is a kernel function, which is defined as $K(x, y)=\exp -\|x-y\|$, i.e., a Gaussian kernel function, in our evaluation.

$X=\left[\begin{array}{c}\phi\left(x_{1}\right)^{T} \\ \vdots \\ \phi\left(x_{N}\right)^{T}\end{array}\right]$

$\bar{X}=\left[\begin{array}{c}{\overline{\phi\left(x_{1}\right)}}^{T} \\ \vdots \\ {\overline{\phi\left(x_{N}\right)}}^{T}\end{array}\right]=\left[\begin{array}{c}1, \phi\left(x_{1}\right)^{T} \\ \vdots \\ 1, \phi\left(x_{N}\right)^{T}\end{array}\right]=\left[1_{N \times 1}, X\right]$

$y=\left[\begin{array}{c}y_{1} \\ \vdots \\ y_{N}\end{array}\right]$

$f(x)=\left(\alpha_{1}+\ldots+\alpha_{N}\right)+\alpha_{1} K\left(x_{1}, x\right)+\ldots+\alpha_{N} K\left(x_{N}, x\right)(19)$

$\alpha=\bar{X}\left(\bar{X}^{T} \bar{X}\right)^{-2} \bar{X}^{T} y$

\subsection{Sampling Selection Methods}

When a regression model is established by a given approximation method, the sample data should be selected with certain criteria to train the model. This is also a subject of study in this paper. We use three sampling data selection methods in our investigation to understand differences of the FWA acceleration performance by these three sample data selection methods. They are listed as follows.

- The best sampling method selects the best $K$ individuals as sampling data.

- The distance near the best fitness individual sampling method selects the nearest $K$ individuals to the best individual using Euclidean distance as sampling data.

- The random sampling method selects $K$ individuals randomly as sampling data.

\subsection{Sampling Sizes}

The sampling size is another factor that influences the approximate model's accuracy and acceleration performance of the FWA. Theoretically, where a large sampling size is applied in the approximation or interpolation process, the better approximation result will be obtained, if over-fitting does not occur. In this study, the influence of sampling size on acceleration performance of the FWA is also a subject of investigation.
Specifically, we set the sample size as 3, 5, 10 for the methods that conduct the approximation and interpolation in lower and higher dimensional space, and set the sampling size as $2 * D+1$ ( $D$ is the dimension of benchmark function) for the approximation and interpolation in original space, because the regression model in original space has $2 * D+1$ unknown parameters.

\subsection{Surrogate-assisted Fireworks Algorithm}

The surrogate-assisted FWA uses an approximation or interpolation model of fitness landscape as the surrogate model to assist FWA search. The approximation or interpolation can be conducted in lower dimensional space, original space and high dimensional space. After we obtain the approximated fitness landscape, we can apply the elite strategy from the approximated fitness landscape to enhance optimization performance of the FWA.

The elite strategy in lower dimensional space for approximating fitness landscape uses only one of the $n$ parameter axes at a time instead of all $n$ parameter axes, and projects individuals onto each 1-dimensional (1-D) space. Each of the $n 1-\mathrm{D}$ spaces has $K$ projected individuals, which come from the different sampling methods with a different sampling size. We approximate the landscape of each 1-D space using the projected $K$ individuals and select the elite from the $n$ approximated 1-D landscape shapes. The elite are generated from the resulting 1-D approximated shapes, see figure 1 .

The actual least square regression functions used is polynomial curve fitting, given by Eq. (21), where $x_{i j}$, $(i=1,2, \ldots, D)$ and $(j=1,2, \ldots, K)$ are the projected individual of point set $X_{i},(i=1,2, \ldots, D)$ and their fitness values among $\left(x_{i j}, y_{j}\right)$ in the $i$-th 1 -D regression space for $(i=1,2, \ldots, D)$ and $(j=1,2, . ., K), a_{0}$, $a_{1}, \cdots, a_{k}$ are the parameters obtained by least squares method, $t$ is the power of polynomial function.

$\left(\begin{array}{cccc}x_{11}^{t} & x_{12}^{t-1} & \ldots & x_{1 K}^{0} \\ x_{21}^{t} & x_{21}^{t-1} & \ldots & x_{2 K}^{0} \\ \vdots & \vdots & & \vdots \\ x_{D 1}^{t} & x_{D 2}^{t-1} & \ldots & x_{D K}^{0}\end{array}\right)\left(\begin{array}{c}a_{1} \\ a_{2} \\ \vdots \\ a_{K}\end{array}\right)=\left(\begin{array}{l}y_{1} \\ y_{2} \\ \vdots \\ y_{K}\end{array}\right)$

Least square approximation by a two-degree polynomial function $(t=2)$ simplifies a fitness landscape with a non-linear curve, and it is easy to obtain its inflection point from its gradient, using the inflection point as the elite. Linear least square approximation uses a linear function $(t=1)$ to approximate the fitness landscape. Its gradient is either descent or ascent. A safer approach, taking into account both descent and ascent, is to select the average point of the linear approximation 


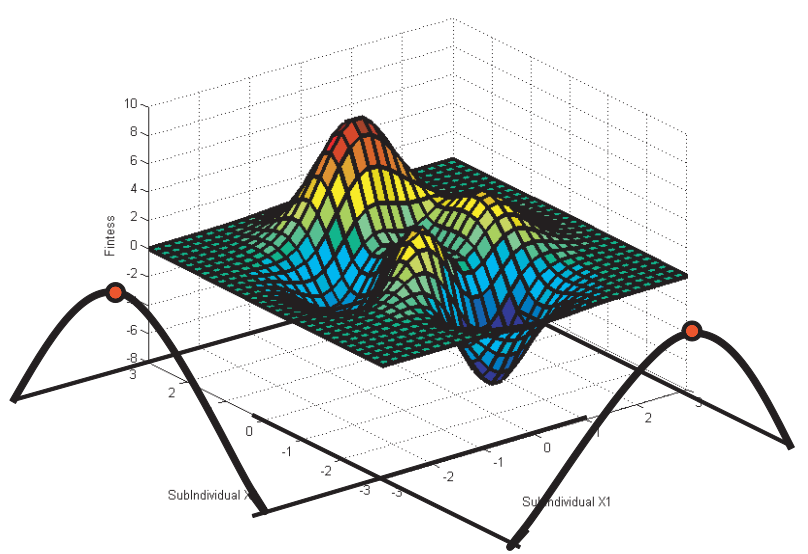

Fig. 1 Original $n$-D space and 1-D spaces obtained by reducing the dimensions of the original one. In our proposed approximation of fitness landscape in lower dimensional space, first, we project fitness landscape in each lower dimension (in this paper, we project it into one dimensional search space, but it is not limited to one dimensional search space), second, we conduct approximation in each lower dimensional search space and obtain elite from each simple shape or surface, finally, we combine these lower dimensional elite together in each related dimensional position as the final elite to enhance FWA optimization performance.

line as the elite. The other approximation and interpolation methods obtain the elite from their shapes by re-sampling 100 times and selecting the re-sample data with the best fitness as the elite.

The proposed methods replace the worst individual in each generation with the selected elite. Although we cannot deny the small possibility that the global optimum is located near the worst individual, the possibility that the worst individual will become a parent in the next generation is also low. Removing the worst individual therefore presents the least risk and is a reasonable choice.

The whole workflow of the surrogate-assisted FWA with an elite strategy is shown in Algorithm 4, where algorithm initialization is in step 2, the optimization of one generation is presented in steps 3 to 17 , and in step 18, the algorithm returns the best obtained solution. It is the crucial process in step 11 to step 16 that explains the elite strategy. This includes (a). obtain sampling data with a certain sampling select method and sampling size from $N$ fireworks (step 11), (b). approximate fitness landscape (step 12), (c). obtain a spark from approximated curves by elite strategy (step 13), and (d). if the fitness of the elite is better than that of the worst firework, the worst one will be replaced (steps 14-16).

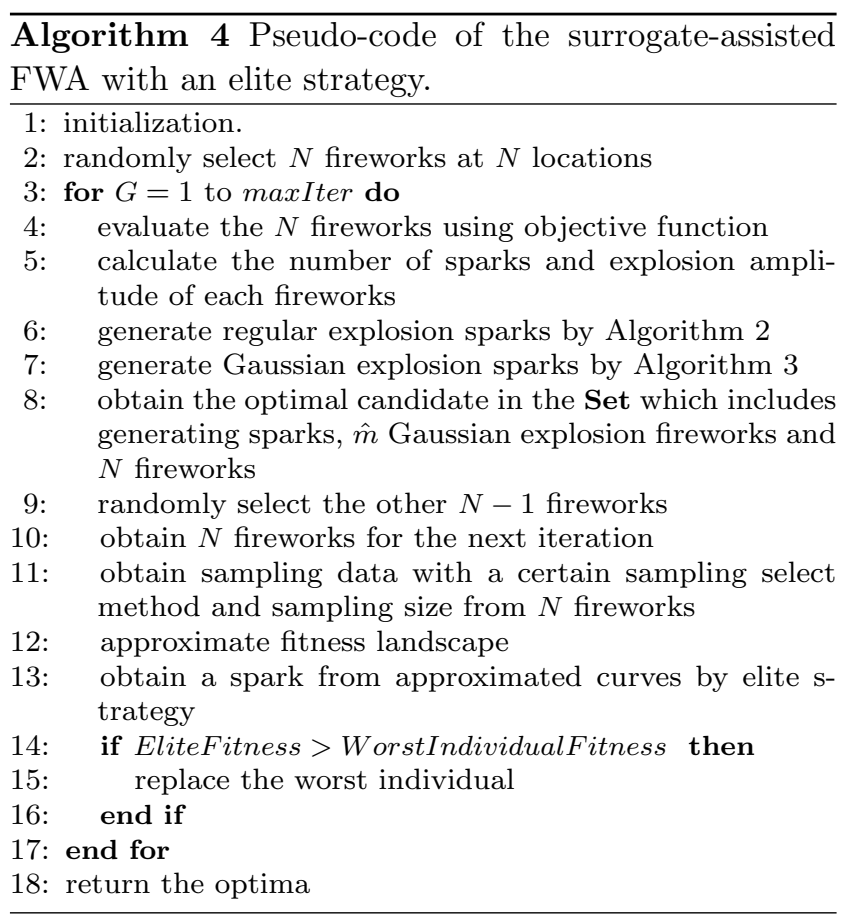

\section{Experimental Evaluation}

\subsection{Experimental Setting}

In our evaluations, the sampling size is set as 3,5 and 10, i.e., $K=3, K=5$ and $K=10$ to investigate the sampling size's influence on the acceleration performance of surrogate-assisted FWA. This sampling size setting is applied in the approximation and interpolation methods in lower and higher dimensional space. It needs at least $2 * D+1$ sampling number for least squares approximation with one and two degree polynomial functions, because there are $2 * D+1$ unknown parameters. We investigate least squares approximation with one and two degree polynomial functions, spline interpolation, Newton interpolation for approximating the fitness landscape in projected 1-D space, and least squares approximation with two degree polynomial functions for approximating fitness landscape in original space, and the SVR with Gaussian kernel function for approximating fitness landscape in high dimensional space. Three sampling selection methods are applied in each approximation and interpolation methods with different sampling sizes.

The number of fireworks $(N)$ and Gaussian explosion firework $(\hat{m})$ are both set as 8 and other parameters are set as in [23]. Experimental evaluations run 30 trials of 1000 generations on each benchmark function independently. The experimental platform is MATLAB 2011b, running under Windows 7 on an Intel Core i7$2600 \mathrm{CPU}$ with $3.7 \mathrm{GHz}$ and 8GB RAM. To validate 
the performance of the proposed algorithm and investigate the influences of different approximation strategies, two groups of experiments are designed: performance comparison on benchmark functions with dimension set to 10 and 30 (10-D and 30-D). The abbreviations of the proposed algorithm, sampling method and sampling size are shown in Table 1.

Table 1 Abbreviation of proposed algorithm, sampling method and sampling size. In this paper, we use six approximation methods, three sampling data selection methods and three sampling sizes in the evaluation experiments. There are total of 48 algorithms plus one canonical FWA.

\begin{tabular}{|c|c|}
\hline abbreviation & meaning \\
\hline LS1 & $\begin{array}{l}\text { least square approximation with linear } \\
\text { function }[12]\end{array}$ \\
\hline LS2 & $\begin{array}{l}\text { least square approximation with second } \\
\text { degree polynomial function }[12]\end{array}$ \\
\hline Spline & Spline interpolation \\
\hline Newton & Newton interpolation \\
\hline OLS & $\begin{array}{l}\text { least square approximation with a single } \\
\text { peak function in original space }[22]\end{array}$ \\
\hline SVR & support vector regression [13] \\
\hline BST & best sampling method \\
\hline DIS & distance sampling method \\
\hline RND & random sampling method \\
\hline 3 & sampling size is 3 \\
\hline 5 & sampling size is 5 \\
\hline 10 & sampling size is 10 \\
\hline
\end{tabular}

\subsection{Benchmark Functions}

We investigate our proposed algorithms and related issues by using benchmark functions from CEC2005 benchmark test suite [20]. In the benchmark functions, 25 functions are included, which presents a variety of fitness landscapes, such as uni-modal and multi-modal, shifted, rotated, global optimum on bounds, etc. Table 2 presents a detailed description of these benchmark functions.

\subsection{Evaluation Metrics}

For comparing and analysing the performance of different algorithms, the fitness values, elite rank, elite location, approximation interval are recorded. The Wilcoxon signed-rank test is applied to validate the significant difference between two algorithms. In our evaluation, the significance level is set to $5 \%$, i.e., $p<0.05$ ( $p$ means p-value in Wilcoxon signed-rank test.).
Table 2 Benchmark functions in our evaluation experiments from [20]. (The abbreviations in this table present as follows. Uni=Uni-modal, Multi=Multi-modal, $\mathrm{Sh}=$ Shifted, $\mathrm{Rt}=$ Rotated, $\mathrm{GB}=$ Global on Bounds, $\mathrm{HC}=$ Hybrid Composition, $\mathrm{NM}=$ Number Matrix)

\begin{tabular}{|l|l|l|l|c|}
\hline No. & Type & Description & Bounds & Optima \\
\hline$f_{1}$ & & Sh Sphere & & -450 \\
$f_{2}$ & & Sh Schwefel 1.2 & & -450 \\
$f_{3}$ & Uni & Sh Rt Elliptic & {$[-100,100]$} & -450 \\
$f_{4}$ & & $f_{2}$ with Noise & & -450 \\
$f_{5}$ & & Schwefel 2.6 GB & & -310 \\
\hline$f_{6}$ & & Sh Rosenbrock & {$[-100,100]$} & 390 \\
$f_{7}$ & & Sh Rt Griewank & {$[0,600]$} & -180 \\
$f_{8}$ & & Sh Rt Ackley GB & {$[-32,32]$} & -140 \\
$f_{9}$ & & Sh Rastrigin & {$[-5,5]$} & -330 \\
$f_{10}$ & Multi & Sh Rt Rastrigin & {$[-5,5]$} & -330 \\
$f_{11}$ & & Sh Rt Weierstrass & {$[-0.5,0.5]$} & 90 \\
$f_{12}$ & & Schwefel 2.13 & {$[\pi, \pi]$} & 460 \\
$f_{13}$ & & Sh Expanded F8F2 & {$[-3,1]$} & -130 \\
$f_{14}$ & & Sh Rt Scaffer F6 & {$[-100,100]$} & -300 \\
\hline$f_{15}$ & & HC Function & & 120 \\
$f_{16}$ & & Rt HC Function 1 & & 120 \\
$f_{17}$ & & $f_{16}$ with Noise & & 120 \\
$f_{18}$ & & Rt HC Function 2 & & 10 \\
$f_{19}$ & & $f_{18}$ with Basin & & 10 \\
$f_{20}$ & Hybrid & $f_{18}$ with GB & {$[-5,5]$} & 10 \\
$f_{21}$ & & Rt HC Funtion 3 & & 360 \\
$f_{22}$ & & $f_{21}$ with NM & & 360 \\
$f_{23}$ & & NC Rt $f_{21}$ & & 360 \\
$f_{24}$ & & Rt HC Function 4 & & 260 \\
$f_{25}$ & & $f_{24}$ without Bounds & & 260 \\
\hline
\end{tabular}

Tables 3 and 4 present the 10-D benchmark function's mean values of each algorithm, and the Wilcoxon signed-rank test results from comparing the canonical FWA and our proposed surrogate-assisted FWA with different approximation methods, different sampling methods and sampling sizes. The $\nmid$ mark in these tables means the proposed method significantly outperforms the canonical FWA by Wilcoxon signed-rank in the significant level $p<0.05$. Table 5 shows the average elite rank from each proposed algorithm for 10$\mathrm{D}$ and 30-D benchmark functions. Figure 2 describes the average times of elite obtained outside of approximation interval, i.e., extrapolation phenomenon occurs. We discuss and analyse optimization performance of each method based on these results.

\section{Discussion}

\subsection{Analysis of Approximation Approach Influence}

In the approximation method aspect, the approximation method in low dimensional space and original space seems to obtain the same optimization results. However, the approximation method in the high dimension space, i.e., support vector regression method, is effective in a few of the benchmark problems. In the project- 
Table 3 Mean value of F1-F14 with 10 dimension. The Abbreviations used here is in Table 1 . The fitness value with $\dagger$ mark presents that this algorithm is significantly better than canonical FWA by Wilcoxon signed-rank in the significant level $p<0.05$.

\begin{tabular}{|c|c|c|c|c|c|c|c|c|c|c|c|c|c|c|}
\hline Method & $f_{1}$ & $f_{2}$ & $f_{3}$ & $f_{4}$ & $f_{5}$ & $f_{6}$ & $f_{7}$ & $f_{8}$ & $f_{9}$ & $f_{10}$ & $f_{11}$ & $f_{12}$ & $f_{13}$ & $f_{14}$ \\
\hline Normal & -442.817 & 194.3666 & 3529551 & 2105.924 & 2656.02 & 2747.469 & -177.339 & -119.899 & -313.709 & -281.939 & 96.29126 & 3093.602 & -128.999 & -296.465 \\
\hline LS1-BST3 & $-449.86 \dagger$ & $-101.5 \dagger$ & $1419161.51 \dagger$ & $132.3 \dagger$ & 1911.9 & $1290.73 \dagger$ & -178.12 & -119.92 & $-323.85 \dagger$ & -287.4 & 96.8 & 1900.35 & $-129.26 \dagger$ & -296.39 \\
\hline LS1-BST5 & $-449.78 \dagger$ & $-165.23 \dagger$ & $1402447.33 \dagger$ & $117.92 \dagger$ & 1610.44 & $1367.29 \dagger$ & -178.24 & -119.91 & $-324.89 \dagger$ & -285.74 & 96.89 & 2028.88 & $-129.32 \dagger$ & -296.44 \\
\hline LS1-BST10 & $-449.76 \dagger$ & $-158.04 \dagger$ & $788825.5 \dagger$ & $43.87 \dagger$ & $1333.34 \dagger$ & $1651.82 \dagger$ & $-178.21 \dagger$ & -119.89 & $-323.52 \dagger$ & -281.69 & $97.3 \dagger$ & $1748.98 \dagger$ & $-129.33 \dagger$ & -296.46 \\
\hline LS1-DIS3 & -436.11 & 59.34 & 3435249 & $524.68 \dagger$ & 2480.33 & 2608.99 & -178.17 & -119.92 & -316.02 & -289.1 & 96.79 & 3092 & -129.08 & -296.43 \\
\hline LS1-DIS5 & -436.49 & $-9.53 \dagger$ & 1970932 & $291.02 \dagger$ & 2468.56 & 3171.58 & -178.18 & -119.9 & -317.74 & -286.67 & 96.33 & 2451.55 & $-129.22 \dagger$ & -296.41 \\
\hline LS1-DIS10 & -442.83 & -31.6 & $1764738.82 \dagger$ & $111.79 \dagger$ & 2569.26 & 2992.26 & -178.05 & -119.9 & -317.82 & -287.97 & 96.07 & 2602.44 & -129.12 & -296.32 \\
\hline LS1-RND3 & $-448.28 \dagger$ & $-271.92 \dagger$ & $858698.8 \dagger$ & $-156.2 \dagger$ & 1731.47 & 2417.13 & $-178.05 \dagger$ & -119.91 & $-320.82 \dagger$ & -288.47 & 96.35 & 2339.96 & $-129.23 \dagger$ & -296.39 \\
\hline LS1-RND5 & $-447.61 \dagger$ & $-279.7 \dagger$ & $975242.14 \dagger$ & $-136.35 \dagger$ & 2737.96 & 3101.7 & $-178.19 \dagger$ & -119.9 & -316.39 & -289.29 & $97.13 \dagger$ & 2738.46 & $-129.23 \dagger$ & -296.42 \\
\hline LS1-RND10 & $-447.13 \dagger$ & $-224.5 \dagger$ & $886462.29 \dagger$ & $-79.06 \dagger$ & 2097.08 & 2393.86 & $-178.27 \dagger$ & -119.9 & -318.63 & -289.4 & 96.49 & 2139.09 & -129.05 & -296.45 \\
\hline LS2-BST3 & $-449.89 \dagger$ & $-257.04 \dagger$ & $1192195.74 \dagger$ & $-15.19 \dagger$ & $965.55 \dagger$ & $1188.36 \dagger$ & $-178.57 \dagger$ & -119.9 & $-324.15 \dagger$ & -286.98 & $97.35 \dagger$ & 2103.86 & $-129.17 \dagger$ & -296.39 \\
\hline LS2-BST5 & $-449.89 \dagger$ & $-215.57 \dagger$ & $1414917.38 \dagger$ & $-83.43 \dagger$ & $1402.14 \dagger$ & 1858.9 & $-178.4 \dagger$ & -119.91 & $-323.94 \dagger$ & -281.64 & $97.24 \dagger$ & 2949.65 & $-129.25 \dagger$ & -296.44 \\
\hline LS2-BST10 & $-449.92 \dagger$ & $-277.6 \dagger$ & $1351030.14 \dagger$ & $-212.34 \dagger$ & $1302.74 \dagger$ & $1314.58 \dagger$ & $-178.48 \dagger$ & -119.91 & $-325.06 \dagger$ & -284.26 & $97.66 \dagger$ & 1913.94 & -129.2 & -296.39 \\
\hline LS2-DIS3 & $-446.63 \dagger$ & 32.59 & $1320490.13 \dagger$ & $289.61 \dagger$ & 1665.86 & 2504.29 & -178.2 & -119.88 & -315.95 & -288.34 & $95.41 \dagger$ & 3715.42 & $-129.19 \dagger$ & -296.48 \\
\hline LS2-DIS5 & -443.47 & $-21.89 \dagger$ & $1277189.38 \dagger$ & $121.5 \dagger$ & 1640.35 & 3104.63 & $-178.43 \dagger$ & -119.9 & -316.51 & $-294.73 \dagger$ & $95.29 \dagger$ & 2721.99 & $-129.27 \dagger$ & -296.39 \\
\hline LS2-DIS10 & -444.23 & $-3.62 \dagger$ & $956602.9 \dagger$ & $93.92 \dagger$ & $1025.44 \dagger$ & 1931.72 & $-178.48 \dagger$ & -119.9 & -317.39 & -291.66 & $94.79 \dagger$ & $1835.85 \dagger$ & -129.21 & -296.44 \\
\hline LS2-RND3 & $-449.94 \dagger$ & $-400.39 \dagger$ & $849730.96 \dagger$ & $-321.26 \dagger$ & $1002.5 \dagger$ & $1681.65 \dagger$ & $-178.83 \dagger$ & -119.87 & $-323.14 \dagger$ & -286.08 & 96.8 & $1596.95 \dagger$ & $-129.3 \dagger$ & -296.47 \\
\hline LS2-RND5 & $-449.91 \dagger$ & $-408.07 \dagger$ & $670058.42 \dagger$ & $-346.44 \dagger$ & $662.76 \dagger$ & 2387.03 & $-178.87 \dagger$ & -119.91 & $-322.68 \dagger$ & $-291.16 \dagger$ & 96.39 & $1610.89 \dagger$ & $-129.24 \dagger$ & -296.5 \\
\hline LS2-RND10 & $-449.29 \dagger$ & $-395.9 \dagger$ & $791469.78 \dagger$ & $-348.92 \dagger$ & $-148.03 \dagger$ & 2784.89 & $-178.77 \dagger$ & -119.91 & $-319.99 \dagger$ & $-293.86 \dagger$ & 96.55 & 2709.44 & $-129.23 \dagger$ & -296.46 \\
\hline Spline-BST3 & $-449.92 \dagger$ & $-247.85 \dagger$ & $1456508.65 \dagger$ & $17.25 \dagger$ & $1357.85 \dagger$ & $1146.91 \dagger$ & -178.3 & -119.93 & $-324.79 \dagger$ & -283.57 & 96.87 & $1329.48 \dagger$ & \begin{tabular}{|l|}
-129.19 \\
\end{tabular} & -296.48 \\
\hline Spline-BST5 & $-449.91 \dagger$ & $-286.57 \dagger$ & $989402.74 \dagger$ & $-83.7 \dagger$ & $1468.55 \dagger$ & $1305.05 \dagger$ & $-178.31 \dagger$ & -119.89 & $-323.28 \dagger$ & -288.39 & 97.14 & $1760.23 \dagger$ & $-129.27 \dagger$ & -296.47 \\
\hline Spline-BST10 & $-449.89 \dagger$ & $-258.16 \dagger$ & $894168.3 \dagger$ & $-140.85 \dagger$ & $941.19 \dagger$ & $1223.47 \dagger$ & $-178.46 \dagger$ & -119.9 & $-323.89 \dagger$ & -287.49 & $97.06 \dagger$ & $1693.48 \dagger$ & $-129.25 \dagger$ & -296.41 \\
\hline Spline-DIS3 & -444.66 & 11.91 & $1215298.95 \dagger$ & $151.95 \dagger$ & 2173.85 & 2988.47 & -178.3 & -119.91 & -316.77 & -288.65 & 95.94 & 2265.62 & -129.1 & $-296.31 \dagger$ \\
\hline Spline-DIS5 & $-446.31 \dagger$ & $-27.49 \dagger$ & $1564324.95 \dagger$ & $155.87 \dagger$ & 1397.71 & 3298.73 & -178.01 & -119.9 & -318.4 & -288.17 & 96.14 & 3144.26 & -129.09 & -296.42 \\
\hline Spline-DIS10 & -442 & 189.68 & $2333430.22 \dagger$ & $403.14 \dagger$ & 2520.19 & 3825 & $-178.31 \dagger$ & -119.92 & -317.87 & $-292.67 \dagger$ & 96.43 & & $-129.21 \dagger$ & -296.35 \\
\hline Spline-RND3 & $-449.96 \dagger$ & $-390.02 \dagger$ & $825508.08 \dagger$ & $-269.14 \dagger$ & $1452.06 \dagger$ & 1887.33 & $-178.99 \dagger$ & -119.89 & $-322.37 \dagger$ & $-294.82 \dagger$ & 96.7 & 2227.35 & $-129.23 \dagger$ & -296.46 \\
\hline Spline-RND5 & $-449.04 \dagger$ & $-378 \dagger$ & $912587.82 \dagger$ & $-285.24 \dagger$ & $1068.86 \dagger$ & $1582.92 \dagger$ & -178.29 & -119.9 & $-322.85 \dagger$ & $-293.09 \dagger$ & 96.72 & 2681.79 & $-129.24 \dagger$ & -296.5 \\
\hline Spline-RND10 & $-447.26 \dagger$ & $-315.03 \dagger$ & $1097016.49 \dagger$ & $-138.69 \dagger$ & $1342.57 \dagger$ & 3415.72 & -178.1 & -119.89 & -318.63 & $-297.78 \dagger$ & $97.04 \dagger$ & 3660.93 & -129.13 & -296.48 \\
\hline Newton-BST3 & $-449.92 \dagger$ & $-222.3 \dagger$ & $1088009.58 \dagger$ & $-24.61 \dagger$ & $1320.09 \dagger$ & $951.15 \dagger$ & $-178.28 \dagger$ & -119.93 & $-325.38 \dagger$ & $-290.73 \dagger$ & $97.1 \dagger$ & $1499.62 \dagger$ & -129.18 & -296.37 \\
\hline Newton-BST5 & $-449.88 \dagger$ & $-282.92 \dagger$ & $1302796.27 \dagger$ & $-177.06 \dagger$ & $1219.89 \dagger$ & $1816.16 \dagger$ & $-178.38 \dagger$ & -119.91 & $-324.09 \dagger$ & -283.87 & $96.96 \dagger$ & $1624.2 \dagger$ & $-129.23 \dagger$ & $-296.34 \dagger$ \\
\hline Newton-BST10 & $-449.91 \dagger$ & $-269.11 \dagger$ & $1043788.28 \dagger$ & $-192.28 \dagger$ & $1037.99 \dagger$ & $1046.51 \dagger$ & $-178.48 \dagger$ & & $-324.59 \dagger$ & & & & & -296.47 \\
\hline Newton-DIS3 & -432.54 & $-48.45 \dagger$ & $926684.82 \dagger$ & $182.46 \dagger$ & 2591.17 & 2205.05 & -177.98 & -119.91 & -317.26 & -288.24 & $95.43 \dagger$ & 2699.62 & $-129.25 \dagger$ & -296.4 \\
\hline Newton-DIS5 & -440.33 & $-5.52 \dagger$ & $1892874.1 \dagger$ & $400.36 \dagger$ & 2809.02 & 20998.53 & -177.74 & & -314.79 & -291.19 & 96.1 & & & -296.51 \\
\hline Newton-DIS10 & -442.37 & 64.79 & 2570233 & $507.12 \dagger$ & $1169.78 \dagger$ & 3362.79 & -177.25 & -119.9 & -314.81 & $-291.8 \dagger$ & 96.53 & 2857.18 & -129.15 & $-296.31 \dagger$ \\
\hline Newton-RND3 & $-449.97 \dagger$ & $-389.88 \dagger$ & $569467.54 \dagger$ & $-305.42 \dagger$ & 1524.2 & $1318.48 \dagger$ & $-178.5 \dagger$ & -119.9 & $-322.91 \dagger$ & -285.49 & 96.39 & $1492.15 \dagger$ & -129.19 & -296.57 \\
\hline Newton-RND5 & $-448.15 \dagger$ & $-313.72 \dagger$ & $1050597.74 \dagger$ & $-199.77 \dagger$ & 1385.18 & 2467.76 & $-178.47 \dagger$ & -119.89 & -318.66 & $-293.95 \dagger$ & 96.71 & 1937.32 & $-129.24 \dagger$ & -296.47 \\
\hline Newton-RND10 & -443.95 & $-109.22 \dagger$ & $1613831.5 \dagger$ & $0.6 \dagger$ & 2337.95 & 2973 & -177.39 & & -317.17 & -29 & 96.2 & .04 & -129.12 & -296.51 \\
\hline OLS-BST & $-450 \dagger$ & $559.63 \dagger$ & 3617660 & $1343.82 \dagger$ & 2393.34 & 3948.39 & -177.58 & -119.9 & -312.86 & -282.75 & $97.28 \dagger$ & 4286.03 & -128.92 & $-296.31 \dagger$ \\
\hline OLS-DIS & $-450 \dagger$ & 538.24 & 3539541 & 1867.47 & 2305.32 & 3983.74 & -177.6 & -119.89 & -314.52 & -281.25 & $97.6 \dagger$ & $4359.67 \dagger$ & -128.84 & $-296.32 \dagger$ \\
\hline OLS-RND & $-450 \dagger$ & 376.47 & 3837140 & 1652.45 & 2408.79 & 15393.14 & -177.86 & -119.9 & -313.87 & -282.28 & $97.44 \dagger$ & 3265.02 & -128.84 & -296.4 \\
\hline SVR-BST3 & -426.64 & 91.3 & 4065658 & $790.6 \dagger$ & 2458.76 & 5481.64 & -177.02 & -119.89 & -313.73 & -282.11 & $97.21 \dagger$ & 3562.28 & -128.87 & -296.42 \\
\hline SVR-BST5 & -441.55 & 99.95 & 2990677 & $809.31 \dagger$ & 2509.09 & 2112.06 & -177.81 & -119.89 & -316.18 & -274.96 & $97.19 \dagger$ & 2761.54 & -128.89 & $-296.26 \dagger$ \\
\hline SVR-BST10 & -442.19 & 187.68 & 3023460 & $738.06 \dagger$ & 2057.05 & 3348.84 & -176.97 & -119.89 & -315.45 & -285.85 & $97.37 \dagger$ & 2798.39 & -128.79 & $-296.29 \dagger$ \\
\hline SVR-DIS3 & -444.15 & 237.32 & 4064241 & $941.43 \dagger$ & 2055.91 & 20456.17 & -176.72 & -119.9 & -315.24 & -287.89 & $97.42 \dagger$ & 3037.76 & -128.85 & -296.34 \\
\hline SVR-DIS5 & -439.18 & 263.48 & 3503445 & $831.27 \dagger$ & 2473.14 & 2846.32 & -177.14 & -119.9 & -311.29 & -283.09 & $97.56 \dagger$ & 3899.99 & -128.91 & $-296.22 \dagger$ \\
\hline SVR-DIS10 & -423.74 & 420.05 & 4459042 & $823.04 \dagger$ & 2715.31 & 3491.04 & -177.56 & -119.92 & -312.53 & -284.94 & $97.22 \dagger$ & 3449.06 & -128.93 & -296.36 \\
\hline SVR-RND3 & -442.46 & 311.95 & 3303740 & $734.16 \dagger$ & 1900.99 & 2632.49 & -177.13 & -119.89 & -310.99 & -283.3 & $97.06 \dagger$ & 3149.49 & -128.94 & $-296.31 \dagger$ \\
\hline SVR-RND5 & -443.38 & 227.1 & 3920565 & $838.7 \dagger$ & 2308.59 & 2039.71 & -177.65 & -119.89 & -315.4 & -284.39 & $97.39 \dagger$ & 3265.26 & -128.91 & $-296.3 \dagger$ \\
\hline SVR-RND10 & -440.78 & 399.52 & 2876402 & $785.14 \dagger$ & 2315.02 & 2544.44 & -177.54 & -119.9 & -309.91 & -284.97 & $97.21 \dagger$ & 3501.03 & -128.87 & -296.42 \\
\hline
\end{tabular}

ed high dimension feature space, linear regression can be perfectly constructed by the SVR method, however, this model cannot present the actual fitness landscape in the original space. The over-fitting issue is the reason that leads to the worse fitness approximation and optimization performance, so, from a practical viewpoint, the SVR is not a good approximation method that can be applied in a fitness landscape approximation field. The same results are also obtained in the work of [13].

Compared with approximation methods in lower dimensional space and that in original space, optimization results by lower dimensional approximation are better than that by original dimensional approximation from the observations of Tables 3 and 4 . This indicates that rough and simple approximation of fitness landscape can be useful in EC enhancement application, and the exact approximation performance sometimes leads to local optimal. Approximation in original space needs more time because of many matrix operations, its computational complexity is higher than that of approximation in lower dimension space. Along with the dimension increasing, the computational complexity of approximation in original space will dramatically rise up. Comparing these two methods applied in 10-D and 30-D benchmark problems, optimization performance obtained by approximation in low dimensional space outperforms that obtained by approximation in original space. As dimensionality is more higher, the optimization result is more better. So, from whatever theoretical analysis and practical evaluation of these two approximation method, approximation in low dimension by dimension reduction technique has its advantages and practical benefits.

Our proposed methods fail to optimize the complex fitness landscape benchmark function (e.g., $f_{18}$, $f_{19}$, and $f_{20}$ ) and specific fitness landscape structure (e.g., $f_{8}$ : optimum at bounds, $f_{12}$ : shifted global optimum, etc.). This result indicates a limitation of our proposed methods, which is effective for the problems with simple fitness landscape. We should analyze the characteristics of the optimization problem and select a proper approximation method to obtain the better optimization performance before we apply our proposed surrogate-assisted FWA. 
Table 4 Mean value of F15-F25 with 10 dimension. The Abbreviations used here are as in Table 1 . The fitness value with $\dagger$ mark presents that this algorithm is significantly better than the canonical FWA by Wilcoxon signed-rank in the significant level $p<0.05$.

\begin{tabular}{|c|c|c|c|c|c|c|c|c|c|c|c|}
\hline Method & $f_{15}$ & $f_{16}$ & $f_{17}$ & $f_{18}$ & $f_{19}$ & $f_{20}$ & $f_{21}$ & $f_{22}$ & $f_{23}$ & $f_{24}$ & $f_{25}$ \\
\hline Normal & 340.16 & 354.47 & 367.22 & 910 & 899.05 & 910 & 145.75 & 1312.65 & 1465.55 & 1266.35 & 1233.37 \\
\hline LS1-BST3 & 357.54 & $315.17 \dagger$ & 358.34 & 903.36 & 910 & 910 & 1334.14 & 1287.47 & 1347.34 & 1195.87 & 1211.92 \\
\hline LS1-BST5 & 344.51 & $309.48 \dagger$ & 339.62 & 910 & 910 & 910 & $1265.84 \dagger$ & $1240.96 \dagger$ & 1411.41 & 1171.43 & 1243.55 \\
\hline LS1-BST10 & $262.14 \dagger$ & $332.2 \dagger$ & 335.6 & 910 & 910 & 906.68 & 1289.92 & $1234.98 \dagger$ & 1341.27 & 1195.16 & 1147.69 \\
\hline LS1-DIS3 & 308.37 & 340.78 & 351.17 & 910 & 910 & 906.69 & 1294.18 & $1273.91 \dagger$ & 1386.8 & 1209.28 & 1032.63 \\
\hline LS1-DIS5 & 319.88 & $318.58 \dagger$ & 356.68 & 910 & 910 & 910 & 1388.08 & $1247.67 \dagger$ & 1458.21 & 1164.79 & 1044.72 \\
\hline LS1-DIS10 & 334.73 & $321.64 \dagger$ & 345.82 & 910 & 899.25 & 910 & 1341.51 & $1225.36 \dagger$ & $1292.33 \dagger$ & 1091.6 & 1027.73 \\
\hline LS1-RND3 & 397.81 & $306.97 \dagger$ & 345.66 & 910 & 910 & 910 & $1227.54 \dagger$ & $1226.6 \dagger$ & $1252.72 \dagger$ & 1085.68 & 1194.81 \\
\hline LS1-RND5 & 327.68 & $309.34 \dagger$ & $332.2 \dagger$ & 906.7 & 906.69 & 910 & 1284.35 & $1249.68 \dagger$ & 1358.92 & 1112.71 & 1065.58 \\
\hline LS1-RND10 & 353.65 & $295.95 \dagger$ & 337.04 & 910 & 910 & 910 & 1438.49 & 1255.8 & $1254.12 \dagger$ & $930.92 \dagger$ & 1215.71 \\
\hline LS2-BST3 & 391.73 & $314.24 \dagger$ & 353.79 & 907.31 & 910 & 910 & $1276.67 \dagger$ & 1268.75 & $1283.6 \dagger$ & $985.34 \dagger$ & $922.65 \dagger$ \\
\hline LS2-BST5 & 324.93 & $320.25 \dagger$ & $323.63 \dagger$ & 910 & 910 & 902.68 & 1335.73 & 1279.87 & $1246.34 \dagger$ & $891.72 \dagger$ & 1043.86 \\
\hline LS2-BST10 & 331.02 & $324.96 \dagger$ & 345.05 & 910 & 910 & 910 & 1437.01 & $1270.78 \dagger$ & $1185.12 \dagger$ & $862.58 \dagger$ & $786.74 \dagger$ \\
\hline LS2-DIS3 & 306.85 & $317.02 \dagger$ & $319.9 \dagger$ & 910 & 910 & 910 & 1338.18 & $1224.89 \dagger$ & $1307.18 \dagger$ & $754.94 \dagger$ & $859.67 \dagger$ \\
\hline LS2-DIS5 & 342.62 & $301.65 \dagger$ & $324.21 \dagger$ & 910 & 910 & 910 & $1314.91 \dagger$ & $1215.73 \dagger$ & $1341.27 \dagger$ & $712.9 \dagger$ & $632.18 \dagger$ \\
\hline LS2-DIS10 & 376.87 & $295.44 \dagger$ & $308.5 \dagger$ & 903.45 & 910 & 910 & $1137.49 \dagger$ & $1131.81 \dagger$ & $1236.72 \dagger$ & $642.33 \dagger$ & $580.64 \dagger$ \\
\hline LS2-RND3 & 348.45 & $306.21 \dagger$ & $305.55 \dagger$ & 906.67 & 910 & 906.67 & 1292.36 & $1228.29 \dagger$ & $1323.84 \dagger$ & $903.38 \dagger$ & $839.89 \dagger$ \\
\hline LS2-RND5 & 339.32 & $302.14 \dagger$ & $310.4 \dagger$ & 908.17 & 910 & 894.02 & 1331.99 & $1212.39 \dagger$ & $1323.47 \dagger$ & $801.76 \dagger$ & $841.93 \dagger$ \\
\hline LS2-RND10 & 411.44 & $293.62 \dagger$ & $318.24 \dagger$ & 906.68 & 906.69 & 906.68 & $1204.18 \dagger$ & $1178.71 \dagger$ & $1313.45 \dagger$ & $771.28 \dagger$ & $735.21 \dagger$ \\
\hline Spline-BST3 & 360.71 & $329.85 \dagger$ & 340.09 & 892.53 & 910 & 910 & 1352.12 & $1228.52 \dagger$ & $1255.02 \dagger$ & $983.17 \dagger$ & 1006.88 \\
\hline Spline-BST5 & 347.33 & $308.89 \dagger$ & 339.53 & 910 & 910 & 910 & 1345.99 & 1257.68 & $1288.41 \dagger$ & $710.01 \dagger$ & $703.95 \dagger$ \\
\hline Spline-BST10 & 332.89 & $325.3 \dagger$ & $331.46 \dagger$ & 910 & 910 & 906.69 & 1360.34 & $1257.97 \dagger$ & $1232.96 \dagger$ & $592.28 \dagger$ & $664.01 \dagger$ \\
\hline Spline-DIS3 & 308.51 & $307.12 \dagger$ & $321.01 \dagger$ & 910 & 897.54 & 910 & $1204.07 \dagger$ & $1225.84 \dagger$ & 1355.62 & $550.51 \dagger$ & $696.96 \dagger$ \\
\hline Spline-DIS5 & 269.16 & $306.49 \dagger$ & $317.29 \dagger$ & 910 & 901.41 & 910 & 1373.23 & $1174.35 \dagger$ & 1383.61 & $602.23 \dagger$ & $552.81 \dagger$ \\
\hline Spline-DIS10 & 322.63 & $299.11 \dagger$ & $326.97 \dagger$ & 910 & 906.8 & 910 & 1347.24 & $1228.09 \dagger$ & $1248.46 \dagger$ & $586.59 \dagger$ & $484.93 \dagger$ \\
\hline Spline-RND3 & 376.83 & $302.59 \dagger$ & $322.68 \dagger$ & 900.02 & 906.67 & 910 & 1301.39 & $1250.29 \dagger$ & $1245.65 \dagger$ & $789.09 \dagger$ & $790.49 \dagger$ \\
\hline Spline-RND5 & 307.3 & $292.15 \dagger$ & $310.09 \dagger$ & 905.16 & 910 & 892.54 & 1444.25 & $1239.6 \dagger$ & $1245.14 \dagger$ & $678.41 \dagger$ & $694.62 \dagger$ \\
\hline Spline-RND10 & 350.39 & $281.49 \dagger$ & $293.82 \dagger$ & 896.52 & 910 & 906.7 & $1192.21 \dagger$ & $1203.38 \dagger$ & $1229.96 \dagger$ & $533.59 \dagger$ & $554.19 \dagger$ \\
\hline Newton-BST3 & 336.2 & $320.26 \dagger$ & 345.13 & 897.39 & 910 & 910 & 1394.47 & 1275.63 & $1260.61 \dagger$ & $961.87 \dagger$ & $814.9 \dagger$ \\
\hline Newton-BST5 & 346.33 & $316.93 \dagger$ & 359.37 & 908.92 & 906.68 & 910 & 1363.35 & $1234.88 \dagger$ & $1302.64 \dagger$ & $793.32 \dagger$ & 1039.07 \\
\hline Newton-BST10 & 373.71 & $315.97 \dagger$ & 350.54 & 910 & 910 & 910 & 1285.76 & $1249.44 \dagger$ & $1290.51 \dagger$ & $537.27 \dagger$ & $533.59 \dagger$ \\
\hline Newton-DIS3 & 344.12 & $292.97 \dagger$ & $322.62 \dagger$ & 910 & 910 & 900.19 & 1331.69 & $1172 \dagger$ & 1348.93 & $554.34 \dagger$ & $779.32 \dagger$ \\
\hline Newton-DIS5 & 293.21 & $300.04 \dagger$ & $317.9 \dagger$ & 910 & 910 & 910 & 1374.93 & $1224.84 \dagger$ & $1252.63 \dagger$ & $644.01 \dagger$ & $799.26 \dagger$ \\
\hline Newton-DIS10 & 263.68 & $309.35 \dagger$ & $319.67 \dagger$ & 910 & 910 & 910 & 1323.63 & $1259.07 \dagger$ & 1329.89 & $634.88 \dagger$ & $702.7 \dagger$ \\
\hline Newton-RND3 & 359.21 & $307.62 \dagger$ & $322.73 \dagger$ & 910 & 910 & 890.43 & $1243.67 \dagger$ & $1211.18 \dagger$ & $1303.19 \dagger$ & $748.81 \dagger$ & $666.79 \dagger$ \\
\hline Newton-RND5 & 346.64 & $298.06 \dagger$ & $314.96 \dagger$ & 898.75 & 900.07 & 910 & 1349.1 & $1189.05 \dagger$ & $1282.72 \dagger$ & $961.56 \dagger$ & $809.37 \dagger$ \\
\hline Newton-RND10 & 384.83 & $281.32 \dagger$ & $302.47 \dagger$ & 910 & 910 & 910 & $1227.61 \dagger$ & $1214.41 \dagger$ & $1289.92 \dagger$ & $730.17 \dagger$ & $918.82 \dagger$ \\
\hline OLS-BST & 323.65 & $334.42 \dagger$ & 373.7 & 910 & 910 & 910 & 1506.18 & 1310.7 & 1464.12 & 1174.51 & 1205.43 \\
\hline OLS-DIS & 418.33 & 342.6 & 354.52 & 910 & 910 & 910 & 1438.71 & 1299.62 & 1427.72 & $1038.17 \dagger$ & 1101.28 \\
\hline OLS-RND & 337.8 & $338.82 \dagger$ & 381.78 & 910 & 910 & 910 & 1537.26 & 1290.63 & 1438.77 & 1183.43 & 1264.36 \\
\hline SVR-BST3 & 338.64 & 339.07 & 352.95 & 910 & 910 & 910 & 1492.54 & 1338.57 & 1417.2 & 1375.7 & 1340.49 \\
\hline SVR-BST5 & 362.44 & $326.14 \dagger$ & 380.58 & 910 & 910 & 910 & 1419.81 & 1323.67 & 1410.95 & 1414.75 & 1089.03 \\
\hline SVR-BST10 & 357.54 & 357.94 & 358.49 & 910 & 910 & 910 & 1445.33 & 1337.11 & 1400.47 & 1250.93 & 1175.33 \\
\hline SVR-DIS3 & 304.78 & 357.39 & 381.44 & 910 & 910 & 910 & 1459.13 & 1326.04 & $1608.32 \dagger$ & 1333.63 & 1193.76 \\
\hline SVR-DIS5 & 357.54 & 359.95 & 360.65 & 910 & 910 & 910 & 1469.35 & 1300.15 & 1502.86 & 1233.33 & 1345.24 \\
\hline SVR-DIS10 & 357.54 & 361.12 & 346.53 & 910 & 910 & 907.12 & 1531.2 & 1304.61 & 1425.09 & 1201.96 & 1188.03 \\
\hline SVR-RND3 & 326.5 & 341.8 & 364.48 & 910 & 910 & 910 & 1499.58 & 1299.03 & 1482.22 & $963.23 \dagger$ & 1277.5 \\
\hline SVR-RND5 & 357.54 & 335.69 & 362.71 & 910 & 904.7 & 910 & 1487.42 & 1310.99 & 1430.69 & 1185.11 & 1336.99 \\
\hline SVR-RND10 & 357.54 & 341.91 & 371.05 & 910 & 910 & 910 & 1336.42 & 1315.77 & 1347.34 & $1078.39 \dagger$ & 1214.85 \\
\hline
\end{tabular}

\subsection{Analysis of Sampling Method Influence}

In this work, we propose three sampling data selection methods, i.e., the BST method, the DIS method, and the RND method, which mean selecting sampling data from the best $n$ data, selecting sampling data from $n$ nearest data from the best data, and selecting sampling data randomly, respectively. From the optimization results by comparing these three methods, when the BST and RND methods can obtain significant optimization performance, the DIS method cannot obtain the same result. From this observation, the DIS method seem$\mathrm{s}$ less useful than other two methods. Sampling data distribution is a significant factor that influences approximation effect. The DIS method selects data that are nearest to the current best point, it leads to local approximation rather than global approximation that can be obtained by the other two methods. This maybe a reason that why the DIS method fails in most of the benchmark problems.

Both the DIS method and BST method need to use search and sorting algorithms for selection. However, the RND method processes the sampling data randomly with a certain distribution probability. From the practical viewpoint, the computational complexity of the RND method is less than that of other two methods, and the RND method can obtain significantly better optimization performance from the above observation and analysis, we conclude that the RND method is a practical sampling selection method that can be reasonably applied in the real world conditions. 
Table 5 Average elite rank of 10-D and 30-D benchmark function when generation $(G)$ equals to 10,100 and 1000 . There are 8 individuals in our evaluation experiments, the number in this table shows the average rank of elite according to its fitness value. The rank with bold font presents the winner algorithms in each generation of $10-\mathrm{D}$ and $30-\mathrm{D}$ benchmark functions.

\begin{tabular}{|c|c|c|c|c|c|c|}
\hline Method & \multicolumn{3}{|c|}{$10-\mathrm{D}$} & \multicolumn{3}{|c|}{$30-\mathrm{D}$} \\
\hline & $\mathrm{G}=10$ & $\mathrm{G}=100$ & $\mathrm{G}=1000$ & $\mathrm{G}=10$ & $\mathrm{G}=100$ & $\mathrm{G}=1000$ \\
\hline LS1-BST3 & 3.46 & 3.59 & 4.03 & 4.21 & 4.89 & 5.34 \\
\hline LS1-BST5 & 2.48 & 2.39 & 2.32 & 2.84 & 2.64 & 2.67 \\
\hline LS1-BST10 & 2.68 & 2.30 & 2.24 & 2.55 & 2.27 & 2.27 \\
\hline LS1-DIS3 & 6.96 & 7.29 & 7.51 & 6.81 & 7.59 & 7.61 \\
\hline LS1-DIS5 & 6.35 & 6.49 & 6.53 & 6.29 & 6.58 & 6.61 \\
\hline LS1-DIS10 & 6.09 & 6.12 & 6.18 & 5.73 & 5.76 & 6.02 \\
\hline LS1-RND3 & 5.23 & 5.07 & 5.04 & 5.36 & 5.05 & 5.07 \\
\hline LS1-RND5 & 5.29 & 4.96 & 5.14 & 5.12 & 5.10 & 5.05 \\
\hline LS1-RND10 & 5.21 & 5.29 & 5.24 & 5.05 & 5.19 & 5.13 \\
\hline LS2-BST3 & 2.19 & 1.94 & 1.96 & 2.26 & 1.99 & 1.97 \\
\hline LS2-BST5 & 2.14 & 1.88 & 1.90 & 2.15 & 1.99 & 1.92 \\
\hline LS2-BST10 & 2.11 & 1.91 & 1.91 & 2.21 & 1.97 & 1.90 \\
\hline LS2-DIS3 & 5.90 & 5.72 & 5.78 & 5.60 & 5.70 & 5.69 \\
\hline LS2-DIS5 & 5.42 & 5.31 & 5.54 & 4.94 & 5.12 & 5.37 \\
\hline LS2-DIS10 & 4.87 & 5.37 & 5.34 & 4.47 & 4.89 & 4.98 \\
\hline LS2-RND3 & 4.49 & 4.13 & 4.10 & 4.33 & 4.36 & 4.31 \\
\hline LS2-RND5 & 4.49 & 4.30 & 4.45 & 4.32 & 4.48 & 4.58 \\
\hline LS2-RND10 & 4.71 & 4.94 & 5.02 & 4.36 & 4.76 & 4.97 \\
\hline Spline-BST3 & 2.11 & 2.03 & 2.06 & 2.17 & 2.06 & 2.01 \\
\hline Spline-BST5 & 2.32 & 2.13 & 2.10 & 2.51 & 2.19 & 2.14 \\
\hline Spline-BST10 & 2.66 & 2.26 & 2.31 & 2.61 & 2.30 & 2.26 \\
\hline Spline-DIS3 & 5.68 & 5.51 & 5.64 & 5.33 & 5.33 & 5.43 \\
\hline Spline-DIS5 & 6.02 & 5.82 & 6.01 & 5.69 & 5.79 & 5.89 \\
\hline Spline-DIS10 & 6.30 & 6.26 & 6.34 & 5.98 & 6.12 & 6.25 \\
\hline Spline-RND3 & 4.49 & 4.05 & 4.07 & 4.29 & 4.21 & 4.18 \\
\hline Spline-RND5 & 5.19 & 5.19 & 5.21 & 5.05 & 5.19 & 5.15 \\
\hline Spline-RND10 & 5.95 & 5.91 & 5.95 & 5.84 & 5.98 & 5.90 \\
\hline Newton-BST3 & 2.19 & 2.00 & 2.07 & 2.12 & 2.06 & 2.03 \\
\hline Newton-BST5 & 2.43 & 2.11 & 2.16 & 2.34 & 2.09 & 2.11 \\
\hline Newton-BST10 & 2.58 & 2.27 & 2.41 & 2.60 & 2.38 & 2.34 \\
\hline Newton-DIS3 & 5.67 & 5.56 & 5.66 & 5.39 & 5.28 & 5.58 \\
\hline Newton-DIS5 & 6.11 & 6.07 & 6.06 & 5.70 & 5.87 & 6.00 \\
\hline Newton-DIS10 & 6.45 & 6.48 & 6.63 & 6.29 & 6.51 & 6.48 \\
\hline Newton-RND3 & 4.48 & 4.18 & 4.08 & 4.31 & 4.35 & 4.24 \\
\hline Newton-RND5 & 5.59 & 5.55 & 5.55 & 5.50 & 5.62 & 5.47 \\
\hline Newton-RND10 & 6.56 & 6.62 & 6.71 & 6.49 & 6.59 & 6.57 \\
\hline OLS-BST & 5.65 & 5.54 & 5.43 & 7.25 & 6.37 & 6.33 \\
\hline OLS-DIS & 7.02 & 6.95 & 6.75 & 7.29 & 6.56 & 6.36 \\
\hline OLS-RND & 6.96 & 6.74 & 6.62 & 7.51 & 6.93 & 6.96 \\
\hline SVR-BST3 & 7.58 & 7.21 & 7.16 & 7.21 & 7.05 & 7.09 \\
\hline SVR-BST5 & 7.51 & 7.13 & 7.21 & 6.91 & 6.69 & 6.69 \\
\hline SVR-BST10 & 7.36 & 6.75 & 6.73 & 6.99 & 6.71 & 6.76 \\
\hline SVR-DIS3 & 7.62 & 7.17 & 7.13 & 7.29 & 7.09 & 7.05 \\
\hline SVR-DIS5 & 7.46 & 6.78 & 6.74 & 7.02 & 6.67 & 6.73 \\
\hline SVR-DIS10 & 7.55 & 6.70 & 6.77 & 7.14 & 6.67 & 6.72 \\
\hline SVR-RND3 & 8.05 & 7.56 & 7.69 & 7.63 & 7.43 & 7.47 \\
\hline SVR-RND5 & 7.72 & 7.33 & 7.30 & 7.30 & 7.12 & 7.18 \\
\hline SVR-RND10 & 7.06 & 6.84 & 6.75 & 7.01 & 6.97 & 7.03 \\
\hline
\end{tabular}

\subsection{Analysis of Sampling Size Influence}

Theoretical speaking, a large sampling size means more accuracy in approximation performance, except over fitting issues. From the evaluation results (i.e., Tables 3 and 4 ), we can obtain the same conclusion by comparing the same approximation method with different sampling sizes. However, more sampling size requires more time spent in the approximation process. When we apply one of the approximation methods with a certain sampling size mentioned in this paper, we should consider the final optimization results and time cost used in approximation process to achieving a balance state for a better optimization performance.

\subsection{Analysis of Obtained Elite Rank}

One characteristic of our proposed method for enhancing the FWA optimization performance is obtaining an elite from simplified approximation fitness landscape. For approximation method in low dimensional space, we obtain elite in each lower dimension space and combine these elite into original space. The fitness rank of obtained elite is one of the evaluation metrics for evaluating performance of our proposed method. Here, we discuss this issue based on Table 5, which shows the average elite rank from our evaluation experiments. From Table 5, the average ranks of elite obtained by the BST method, the RND method and the DIS method are about 2, 5, and 6, respectively. The rank of elite presents the accuracy and performance of each approximate method and each sampling method.

From this average rank result and mean value of each method obtained from Tables 3 and 4, we found that sampling method is an essential factor that influences the optimization performance of our proposed surrogate-assisted FWA. The influence of the sampling method on the optimization performance enhancemen$t$ is more effective than that of sampling size. On the contrary, if the sampling method is inefficient, whatever the sampling size is, the proposed surrogate-assisted FWA cannot be enhanced and improved significantly. This is a new discovery arising from our evaluation experiments.

\subsection{Analysis of Interpolation and Extrapolation of Obtained Elite}

The primary objective of our proposed method is to approximate fitness landscape with a simple shape, so that we can roughly find promising global optimum region to search from this simple shape. From the approximation point of view, the elite is obtained from interpolation or from extrapolation, i.e., the elite is from approximation range or outside of it. This metric is one of the evaluation factors to evaluate approximation performance of our proposal.

Figure 2 presents average extrapolation times of each approximation method applied to 25 benchmark problems. Except for some special cases of approximation in lower dimensional spaces, these methods can obtain the elite from extrapolation, i.e., outside of approximation range, with about 100 times in 1000 generations. Although the elite comes from these methods rarely outside its approximation range, from the evaluation results, these methods can obtain a better optimization performance in most benchmark problems. It indicates that better local search can improve the optimization 


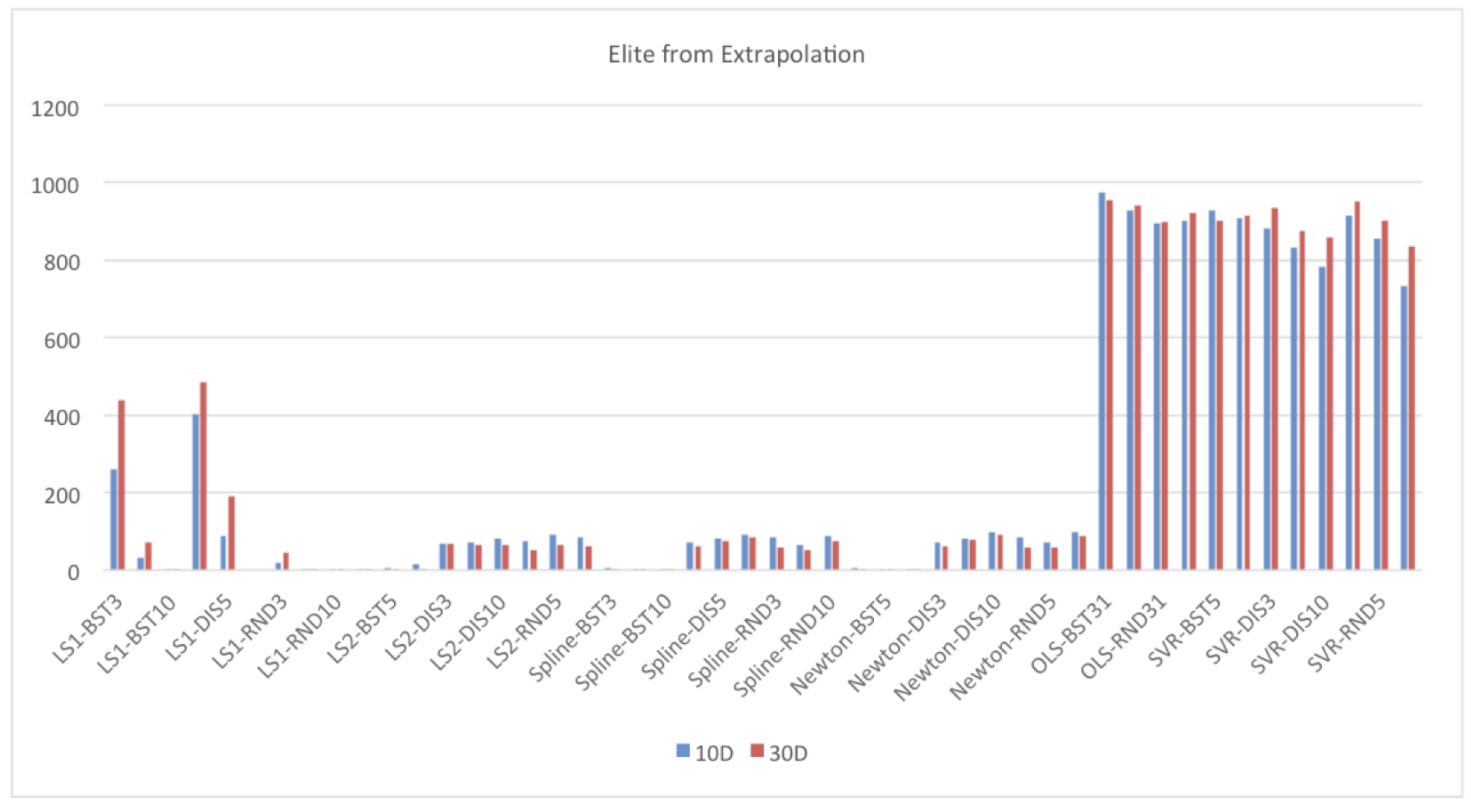

Fig. 2 Extrapolation times of each method, when we obtain the elite from approximated fitness landscape, we judge whether it comes from the interval of sampling data, if it is out of the interval, we define it as extrapolation one time. This figure presents that better local search can improve the optimization performance of FWA. X-axe and Y-axe show the number of average extrapolation times from 25 benchmark functions of each method and each method's abbreviation, respectively.

performance of surrogate-assisted FWA. The times of elite from extrapolation by approximation in original space and high dimension space are more than that of approximation in low dimension. However, as demonstrated in Tables 3 and 4, they cannot present better acceleration performance for most benchmark problems. This result indicates that wrong approximation and over-fitting problem can happen when applying these two types of approximation method.

\section{Conclusion}

In this paper, we investigated and discussed the effectiveness of approximation strategy in surrogate-assisted FWA. We discussed the effectiveness of approximation strategy from the aspect of approximation method, sampling data selection method and sampling size. We analysed and studied the optimization performance of each method. For the approximation method, we use least square approximation, spline interpolation, Newton interpolation, and support vector regression to approximate fitness landscape of FWA in projected lower dimensional, original and higher dimensional search space. We found that approximation in lower dimensional search space can effectively obtain the rough fitness landscape information, and its optimization performance outperforms that of approximation in original and higher dimensional search space. The problem of over-fitting problem frequently happens when applying the SVR method in this fitness landscape approximation problem. In the sampling data selection method aspect, we defined three sampling data selection methods, i.e., the best sampling method, the distance near the best fitness individual sampling method, and the random sampling method to investigate each sampling method's performance. We found that the RND sampling selection method is better than the other two methods from the viewpoints of computational complexity and performance of optimization . From the sample size viewpoint, we set it as 3,5 , and 10 sampling data in each approximation method and sampling selection method. We notice that we should balance the final acceleration performance we obtained and time cost in approximation process we spent. This is a crucial issue when we apply proposed FWA to a variety of problems and real-world applications.

One of the advantages of FWA is that the multiproduction mechanism presents in its algorithm. This means, one firework can generate a number of sparks within the explosion amplitude in each generation. We can use this local fitness information and combine several sparks to explore the global fitness landscape by our approximation method. This is one aspect of our future work. The IEC application requires the optimization algorithm to have a better performance with small number of generations and population size. The fact that 
the FWA can obtain a relatively better optimization performance with a small population size, is another advantage. This characteristic provides an opportunity to establish an interactive framework of FWA, i.e., interactive FWA. Because the evaluation space of humans is relatively simple, we can obtain a better optimization result when we apply our proposed surrogateassisted FWA in interactive FWA by fitness landscape approximation. Some other opportunities for investigation, such as surrogate-assisted FWA selection issue, surrogate-assisted FWA for hard optimization problem$\mathrm{s}$, will be involved in our future work.

\section{Acknowledgement}

This work was supported by the JSPS Grant-in-Aid for Scientific Research (23500279), Japan. This work was also supported by the Natural Science Foundation of China (NSFC) under grant no. 61375119 and 61170057, and partially supported by National Key Basic Research Development Plan (973 Plan) Project of China with grant no. 2015CB352302.

\section{References}

1. Janez Brest, Saso Greiner, Borko Boskovic, Marjan Mernik, and Viljem Zumer. Self-adapting control parameters in differential evolution: A comparative study on numerical benchmark problems. IEEE Transactions on Evolutionary Computation, 10(6):107-125, Feb. 2008.

2. Wenyin Gong, Zhihua Cai, Charles X Ling, and Changhe Li. Enhanced differential evolution with adaptive stategies for numerical optimization. IEEE Transactions on Systems, Man, and Cybernetics, 41(2):397-413, Apr. 2011.

3. Michiel Hazewinkel. Encyclopedia of mathematics, chapter Spline interpolation. Springer, 2001.

4. Andreas Janecek and Ying Tan. Swarm intelligence for non-negative matrix factorization. International Journal of Swarm Intelligence Research (IJSIR), 2(4):12-34, 2011.

5. Yaochu Jin. A comprehensive survey of fitness approximation in evolutionary computation. Soft computing, 9(1):3-12, 2005.

6. Ali Husseinzadeh Kashan. A new metaheuristic for optimization: optics inspired optimization (OIO). Computers E Operations Research, 55:99-125, 2015.

7. Yew-Soon Ong, Meng Hiot Lim, and Xianshun Chen. Research frontier: memetic computation - past, present \& future. IEEE Computational Intelligence Magazine, 5(2):24-36, 2010.

8. Yan Pei. Chaotic evolution: fusion of chaotic ergodicity and evolutionary iteration for optimization. Natural Computing, 13(1):79-96, 2014.

9. Yan Pei. Study on effecient search in evolutionary computation. PhD thesis, Kyushu University, Japan, 2014.

10. Yan Pei. From determinism and probability to chaos: chaotic evolution towards philosophy and methodology of chaotic optimization. The Scientific World Journal, 2015:Article ID 704587, 2015.
11. Yan Pei and Hideyuki Takagi. Fourier analysis of the fitness landscape for evolutionary search acceleration. In 2012 IEEE Congress on Evolutionary Computation (CEC2012), pages 2934-2940, Brisbane, Austrilia, Jun. 2012.

12. Yan Pei and Hideyuki Takagi. Accelerating IEC and EC searches with elite obtained by dimensionality reduction in regression spaces. Journal of Evolutionary Intelligence, 6(1):27-40, 2013.

13. Yan Pei and Hideyuki Takagi. Fitness landscape approximation by adaptive support vector regression with opposition-based learning. In 2013 IEEE International Conference on Systems, Man, and Cybernetics (SMC2013), pages 1329-1334. IEEE, 2013.

14. Yan Pei and Hideyuki Takagi. Triple and quadruple comparison-based interactive differential evolution and differential evolution. In Foundations of Genetic Algorithms Workshop XII (FOGA 2013), number 173-182, Adelaide, Australia, 16-20 Jan. 2013.

15. Yan Pei and Hideyuki Takagi. Local information of fitness landscape obtained by paired comparison-based memetic search for interactive differential evolution. In 2015 IEEE Congress on Evolutionary Computation (CEC2015), pages 2215-2221. IEEE, 2015.

16. Yan Pei and Hiroyuki Takagi. Comparative study on fitness landscape approximation with fourier transform. In 2012 Sixth International Conference on Genetic and Evolutionary Computing (ICGEC2012), pages 400-403. IEEE, 2012.

17. Yan Pei, Shaoqiu Zheng, Ying Tan, and Hideyuki Takagi. An empirical study on influence of approximation approaches to enhance fireworks algorithm. In 2012 IEEE International Conference on Systems, Man, and Cybernetic$s$ (SMC2012), pages 1322-1327, Seoul, Korea, 14-17 Oct. 2012 .

18. A Kai Qin, Vicky Ling Huang, and Ponnuthurai N Suganthan. Differential evolution algorithm with strategy adaptition for global numberical optimization. IEEE Transactions on Evolutionary Computation, 13(2):398-417, Apr. 2009.

19. Ali Sadollah, Hadi Eskandar, Ardeshir Bahreininejad, and Joong Hoon Kim. Water cycle algorithm with evaporation rate for solving constrained and unconstrained optimization problems. Applied Soft Computing, 30:5871, 2015.

20. Ponnuthurai N Suganthan, Nikolaus Hansen, Jing J Liang, Kalyanmoy Deb, YP Chen, Anne Auger, and S Tiwari. Problem definitions and evaluation criteria for the CEC 2005 special session on real-parameter optimization. KanGAL Report, 2005005, 2005.

21. Hideyuki Takagi. Interactive evolutionary computation: fusion of the capabilities of EC optimization and human evaluation. Proceedings of the IEEE, 89(9):1275-1296, 2001.

22. Hideyuki Takagi, Takeo Ingu, and Kei Ohnishi. Accelerating a GA convergence by fitting a single-peak function. Journal of Japan Society for Fuzzy Theory and Intelligent Informatics, 15(2):219-229, 2003. in Japanese.

23. Ying Tan and Yuanchun Zhu. Fireworks algorithm for optimization. In Advances in Swarm Intelligence, pages 355-364. Springer, 2010.

24. Bei Zhang, Min-Xia Zhang, and Yu-Jun Zheng. A hybrid biogeography-based optimization and fireworks algorithm. In Evolutionary Computation (CEC), 2014 IEEE Congress on, pages 3200-3206. IEEE, 2014.

25. Jingqiao Zhang and Arthur C Sanderson. JADE: adaptive differential evolution with external achive. IEEE 
Transactions on Evolutionary Computation, 13(5):945-958, Oct. 2009.

26. Shaoqiu Zheng, Andreas Janecek, and Ying Tan. Enhanced fireworks algorithm. In 2013 IEEE Congress on Evolutionary Computation (CEC2013), pages 2069-2077. IEEE, 2013.

27. Yu-Jun Zheng. Water wave optimization: A new natureinspired metaheuristic. Computers \& Operations Research, 55:1-11, 2015.

28. Yu-Jun Zheng, Qin Song, and Sheng-Yong Chen. Multiobjective fireworks optimization for variable-rate fertilization in oil crop production. Applied Soft Computing, 13(11):4253-4263, 2013. 